\title{
Padé approximations of solitary wave solutions of the Gross-Pitaevskii equation
}

\author{
Natalia G. Berloff \\ Department of Applied Mathematics and Theoretical Physics \\ University of Cambridge, Wilberforce Road, Cambridge, CB3 0WA, UK \\ N.G.Berloff@damtp.cam.ac.uk
}

Submitted 23 June 2003; in press by Journal of Physics A

\begin{abstract}
Padé approximants are used to find approximate vortex solutions of any winding number in the context of Gross-Pitaevskii equation for a uniform condensate and condensates with axisymmetric trapping potentials. Rational function and generalised rational function approximations of axisymmetric solitary waves of the Gross-Pitaevskii equation are obtained in two and three dimensions. These approximations are used to establish a new mechanism of vortex nucleation as a result of solitary wave interactions.
\end{abstract}

Keywords: Gross-Pitaevskii model, Padé approximations, vortex solutions, rarefaction pulses.

\section{Introduction}

In the last decade the experimental realisation of Bose-Einstein condensation in trapped alkali-metal gases at ultralow temperatures has stimulated an intense interest in the production of vortices and vortex arrays and theoretical investigations of their structure, energy, dynamics, and stability [6]. The condensates of alkali vapours are pure and dilute, so that the Gross-Pitaevskii (GP) model which represents the so-called mean-field limit of quantum field theories gives a precise description of the atomic condensates and their dynamics at low temperatures. The same equation has been the subject of extensive studies also in the framework of superfluid helium at very low temperature [5], though the high density and strong repulsive interactions of superfluid helium restrict the applicability of the GP model so that it provides at most a qualitative description.

In spite of the seeming simplicity of the GP model, which is a defocussing nonlinear Schrödinger equation, that has also being extensively studied in other physical systems 
such as nonlinear optics, not many asymptotic or approximate solutions have been found especially for solitary wave solutions in more than one dimensions. Typically one has to resort to numerical integration even in the case of a simple straight line vortex. Other techniques involve power series expansions that have a small radius of convergence and, therefore, are of a very limited use (see more below). The other approach involves quite elaborate asymptotic expansions in several different regions and asymptotic matching between them (19, 2]), which typically yields a useful result for critical parameters of interest such as critical velocities, but the resulting approximation of the solution is too complicated to be used on its own, either in analytic manipulations or as an initial condition (possibly with a perturbation) for numerical calculations. Many numerical studies use a specific vortex configuration as a starting initial condition (see for instance, [13, 14, 16, 3] ) and it is desirable to have a simple approximation to the vortex structure that can be used in such calculations.

The reason for the failure of the power series to represent the vortex solution is that series diverge in the presence of singularities. There are techniques of a summation theory that allow us to overcome this difficulty and represent a given function by a convergent expression. In Euler summation this expression is the limit of a convergent series and in Borel summation this expression is the limit of a convergent integral. But for these methods to work one has to know all the terms of the divergent series exactly before the Euler or Borel sum can be found approximately. Padé summation permits us to use only a few terms of the divergent series to construct an improved estimate of the function.

In what follows we shall modify the standard Padé summation method, so that only the general forms of the power series at zero and at infinity are used to determine the appropriate form of the Padé approximant, but the unknown coefficients will be determined recursively from the GP equation. The general idea is that, if the function $f(x)$ has power series expansions of the form $x^{n} \sum_{i=1}^{\infty} p_{i} x^{2 i-1}$ around $x=0$ and $\sum_{i=0}^{\infty} q_{i} x^{-2 i}$ at infinity, then a Padé approximation of the form

$$
f(x) \approx \sqrt{\frac{x^{2 n} \sum_{i=0}^{N} a_{i} x^{2 i}}{\sum_{j=0}^{N+n} b_{j} x^{2 j}}},
$$

where $a_{N}=q_{0}^{2} b_{N+n}$ with $q_{0}=f(\infty)$ will have the same asymptotics at zero and infinity as the corresponding power series.

Our paper is organised as follows. In Section 2 we will derive a Padé approximation of the straight line vortex in a uniform condensate for any winding number. Sections 3 and 4 deal with vortices in a condensate with trapping potentials including an external potential with a laser beam. In Sections 5 and 6 we develop the rational and generalised rational function approximations of the solitary waves moving with a constant velocity in dimensions two and three correspondingly. In Section 7 we study a new mechanism of vortex nucleation as a result of solitary wave interactions. 


\section{Straight line vortex in a uniform condensate}

We start with the GP model [8, 9] in the form

$$
-2 \mathrm{i} \frac{\partial \psi}{\partial t}=\nabla^{2} \psi+\left(1-|\psi|^{2}\right) \psi
$$

where we use dimensionless variables such that the unit of length corresponds to the healing length $a$, the speed of sound is $c=1 / \sqrt{2}$, and the density at infinity is $\rho_{\infty}=1$. The solution for the straight line vortex $\psi=R(r) \exp (\mathrm{i} n \theta)$ with winding number $n=1,2, \cdots$ in a uniform condensate was first obtained by Pitaevskii [17] via numerical integration of the steady GP equation

$$
R^{\prime \prime}(r)+\frac{1}{r} R^{\prime}(r)-\frac{n^{2}}{r^{2}} R(r)+\left[1-R^{2}(r)\right] R(r)=0,
$$

subject to boundary conditions $R(0)=0$ and $R(\infty)=1$. The asymptotic expansions for small $r$ and large $r$ in terms of the power series were obtained by many authors typically for $n=1$. At small $r$, the solution can be asymptotically approximated by $R(r) \sim$ $\sum_{i=1}^{\infty} p_{i} r^{2 i-1}$, where the first term of the expansion $p_{1}$ has to be determined numerically (by shooting) as $p_{1}=0.5827811878$ with the rest of the terms then generated recursively as $p_{2}=-0.072847648, p_{3}=0.01128249, p_{4}=-0.001781398, \cdots$. The resulting series is useful only within its radius of convergence (i.e., for $r<2.5$, [15]). Asymptotic but divergent solution at infinity can be obtained as $R(r) \sim \sum_{i=0}^{\infty} q_{i} r^{-2 i}$, where $q_{0}=1, q_{1}=-\frac{1}{2}$, $q_{2}=-\frac{9}{8}, \cdots$. In view of the expansion in odd powers at zero and even power expansion at infinity a Padé approximation of the straight line vortex can be obtained in the form (1) with $n=1$ and $N=1$ as

$$
\rho(r)=R(r)^{2}=\frac{r^{2}\left(a_{1}+a_{2} r^{2}\right)}{1+b_{1} r^{2}+b_{2} r^{4}}
$$

where we can let $b_{2}=a_{2}$ in the view of the condition $\rho \rightarrow 1$ as $r \rightarrow \infty$. We substitute the Padé approximation (44) into (3) and expand the resulting expression in series of $r$ setting the coefficient at equal powers of $r$ to zero. At $\mathcal{O}\left(r^{4}\right)$ we get $a_{2}=a_{1}\left(b_{1}-1 / 4\right)$, at $\mathcal{O}\left(r^{6}\right)$ we get $b_{1}=\left(5-32 a_{1}\right) /\left(48-192 a_{1}\right)$, and at $\mathcal{O}\left(r^{8}\right)$ we get $a_{1}=11 / 32$ as the positive root of $11+56 a_{1}-256 a_{1}^{2}=0$. The resulting approximation

$$
R(r) \sim \sqrt{\frac{r^{2}\left(0.3437+0.0286 r^{2}\right)}{1+0.3333 r^{2}+0.0286 r^{4}}}
$$

gives the correct asymptotic behaviour at $r \rightarrow 0$ and at $r \rightarrow \infty$ and approximates the numerical solution very well everywhere. Figure 1 plots the various approximations to the numerically found vortex solution for $n=1$. Note that the procedure described above could be formalised by rescaling both equation (31) and the trial function (44) by $r \rightarrow \epsilon r$, equating 
Figure 1: [Colour online] The plots of the amplitude of the straight vortex line in a uniform condensate as a function of distance from the centre of the vortex. The solid black line gives the solution obtained by numerically integrating (3). Grey line - the Padé approximation (5). Short dashed and long dashed lines give power series expansions at zero (to $\mathcal{O}\left(r^{11}\right)$ ) and at infinity (to $\mathcal{O}\left(r^{-20}\right)$ ) correspondingly.

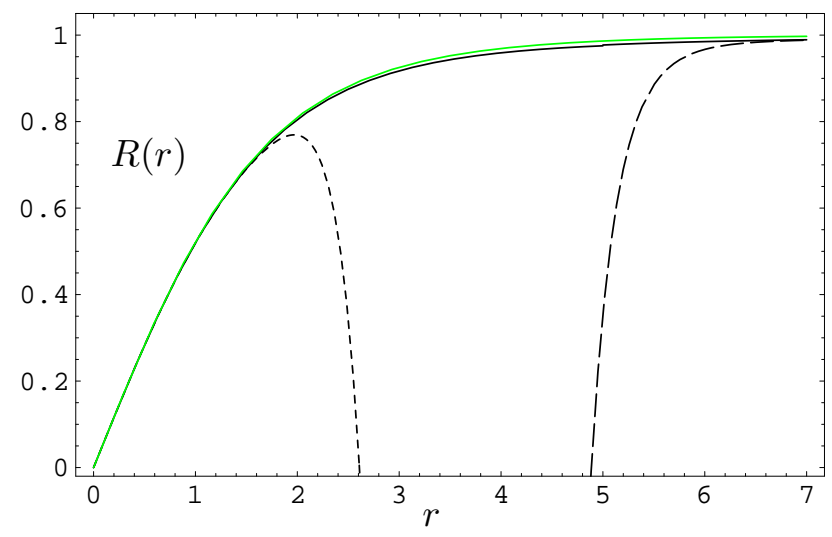

coefficients at powers of $\epsilon$ and setting $\epsilon=1$ (see also [15] for power series expansions). This procedure will become important when we consider solutions involving more than one variable, so that the recursive procedure can be established only by using a different scaling of variables. We could also rewrite the equation (3) in terms of density $\rho=R^{2}(r)$, so that the model equation for the straight line vortex becomes

$$
\frac{d^{2} \rho}{d r^{2}}+\frac{1}{r} \frac{d \rho}{d r}-\frac{1}{2 \rho}\left(\frac{d \rho}{d r}\right)^{2}-\frac{2 \rho n^{2}}{r^{2}}-2 \epsilon^{2}(\rho-1) \rho=0 .
$$

A Padé approximation of the straight line vortex with non unit winding number can be obtained by observing that $R(r) \sim r^{|n|}$ at $r \rightarrow 0$, so that we need to consider the expression

$$
\rho(r) \sim \frac{(\epsilon r)^{2|n|}\left(a_{1}+a_{2}(\epsilon r)^{2}\right)}{\sum_{i=0}^{|n|} b_{i}(\epsilon r)^{2 i}+a_{2}(\epsilon r)^{2|n|+2}}
$$

For instance, for $|n|=2$ we obtained

$$
\rho \sim \frac{r^{4}\left(0.0256+0.0006264 r^{2}\right)}{\left(1+0.19109 r^{2}+0.0196962 r^{4}+0.0006264 r^{6}\right)} .
$$




\section{Straight line vortex in a cigar-like trap}

The equation for the density of a condensate in a cigar-like trap with the vortex trapped in the centre is

$$
\frac{d^{2} \rho}{d r^{2}}+\frac{1}{r} \frac{d \rho}{d r}-\frac{1}{2 \rho}\left(\frac{d \rho}{d r}\right)^{2}-\frac{2 \rho}{r^{2}}-2 \epsilon^{2}\left(\rho-1+\epsilon^{2} \lambda^{2} r^{2}\right) \rho=0
$$

where $\lambda$ is the dimensionless oscillator frequency.

For a noninteracting gas, the condensate wave function for a singly quantised vortex on the symmetry axis involves the first excited radial harmonic oscillator state $\psi_{\text {noninter }} \sim$ $r \exp \left(-\frac{1}{2} \lambda^{2} r^{2}\right) \exp (i \theta)$, so we seek a solution of the form

$$
\rho(r)=\frac{\epsilon^{2} r^{2}\left(a_{1}+a_{2} \epsilon^{2} r^{2}\right)}{1+b_{1} \epsilon^{2} r^{2}+b_{2} \epsilon^{4} r^{4}} \exp \left(-\epsilon^{2} \lambda^{2} r^{2}\right)
$$

where the Taylor expansion will be taken for the exponential function. The expression (10) with $b_{2}=a_{2}$ is substituted into (9) and the terms up to $\mathcal{O}\left(\epsilon^{8}\right)$ are set equal to zero. We get

$$
\begin{aligned}
& a_{2}=\frac{1}{4} a_{1}\left(-1+4 \lambda^{2}+4 b_{1}\right), \\
& b_{1}=-\frac{5+112 \lambda^{4}-48 \lambda^{2}+32 a_{1}\left(6 \lambda^{2}-1\right)}{48\left(4 a_{1}+4 \lambda^{2}-1\right)}
\end{aligned}
$$

with $a_{1}$ given as a positive root of

$$
\begin{aligned}
256 a_{1}^{2}\left(12 \lambda^{2}-1\right) & +\left(4 \lambda^{2}-1\right)^{3}\left(52 \lambda^{2}-11\right) \\
& -8 a_{1}\left(384 \lambda^{6}-528 \lambda^{4}+136 \lambda^{2}-7\right)=0
\end{aligned}
$$

For example, the solution for $\lambda=0.2$ becomes

$$
\rho(r)=\frac{0.2833 r^{2}+0.0136 r^{4}}{1+0.2581 r^{2}+0.0136 r^{4}} \exp \left(-0.04 r^{2}\right)
$$

Similarly to the uniform condensate case, the approximation for the multiply quantised vortices can be found in the form

$$
\rho(r) \sim \frac{(\epsilon r)^{2|n|}\left(a_{1}+a_{2} \epsilon^{2} r^{2}\right)}{\sum_{i=0}^{|n|} b_{i}(\epsilon r)^{2 i}+a_{2}(\epsilon r)^{2|n|+2}} \exp \left[-\epsilon^{2} \lambda^{2} r^{2}\right] .
$$




\section{Vortex in an axisymmetric condensate}

A similar procedure can be implemented to find the vortex in a condensate in an axisymmetric trap given by the external potential $V=\lambda^{2} r^{2}+\lambda_{Z}^{2} z^{2}$. Now, the function $\rho$ depends on two coordinates $\rho=\rho(r, z)$, so that the equation for $\rho$ becomes

$$
\rho_{r r}+\frac{\rho_{r}}{r}-\frac{\rho_{r}^{2}}{2 \rho}-\frac{2 \rho}{r^{2}}+\frac{1}{\epsilon^{2}}\left(\rho_{z z}-\frac{\rho_{z}^{2}}{2 \rho}\right)-2 \epsilon^{2}\left(\rho-1+\epsilon^{2} \lambda^{2} r^{2}+\epsilon^{4} \lambda_{Z}^{2} z^{2}\right) \rho=0,
$$

where we rescaled the variables as $r \rightarrow \epsilon r$ and $z \rightarrow \epsilon^{2} z$. We seek a solution of the form

$$
\rho(r, z)=\frac{\epsilon^{2} r^{2}\left(a_{1}+\epsilon^{2} a_{2} r^{2}\right)}{1+\epsilon^{2} b_{1} r^{2}+\epsilon^{4}\left(b_{2} r^{4}+c_{1} z^{2}\right)} \exp \left(-\epsilon^{2} \lambda^{2} r^{2}-\epsilon^{4} \lambda_{Z}^{2} z^{2}\right) .
$$

We solve the resulting equations to $\mathcal{O}\left(\epsilon^{8}\right)$, define all the parameters in terms of remaining two (say $c_{1}$ and $a_{1}$ ), that are zeros of coupled polynomials, whose roots can be found numerically by fixing $\lambda$ and $\lambda_{Z}$. For instance, for $\lambda=0.2$ and $\lambda_{Z}=0.1$ (and setting $\epsilon=1$ ) we have

$$
\rho=\frac{0.232609 r^{2}+0.0319011 r^{4}}{1+0.342901 r^{2}+0.0319011 r^{4}+0.00697428 z^{2}} \exp \left(-0.04 r^{2}-0.01 z^{2}\right) .
$$

Figure 2 gives the contour plot of this solution in $x z$-plane. The generalisation to multiply charged vortices is carried out in a similar way to previous sections.

The asymptotics of vortices in a trapped condensate was studied by Konotop and Perez-Garcia [12] using a sophisticated multiscale method; their solution was of the form $A \tanh (a r) \exp \left(-b r^{2}-c z^{2}\right)$, where $A, a, b, c$ are some constants that needed to be estimated numerically. The use of a Padé approximation not only provides a swift and easy tool for generating an approximation of comparable accuracy but also has the ability to include a $z$-dependence that is not confined to the exponential term.

There are different ways of creating a vortex in a trapped condensate. In particular, angular momentum can be transmitted to the condensate by rotationally stirring it with a laser beam [4] or by guiding a vortex created at the edge of the condensate by a laser beam towards the centre of condensate [20]. When the vortex is brought to rest at the centre of the condensate, the steady external potential can be assumed to have the form

$$
V(r, z)=\lambda^{2} r^{2}+\lambda_{Z} z^{2}+V_{0} \exp \left[-r^{2} / r_{l}^{2}\right]
$$

where $r_{l}$ is the half-width of the laser beam intensity profile. Our procedure for finding the approximate solution can be automatically adjusted for finding the vortex density in the condensate with potential (19). We seek a Padé approximant (17) as a solution of the 
Figure 2: A density contour plot in the $x z$-plane for a condensate containing a vortex along the $z$-axis as the solution of (16) given by (18). The trap parameters are $\lambda=0.2$ and $\lambda_{Z}=0.1$. Luminosity is proportional to density, the white area being the most dense.

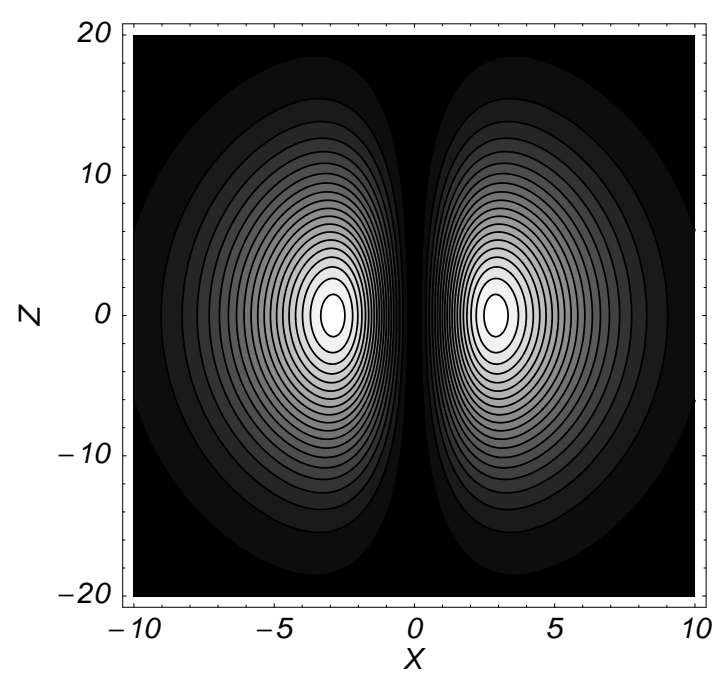

equation

$$
\begin{aligned}
\rho_{r r}+\frac{\rho_{r}}{r}-\frac{\rho_{r}^{2}}{2 \rho} & -\frac{2 \rho}{r^{2}}+\frac{1}{\epsilon^{2}}\left(\rho_{z z}-\frac{\rho_{z}^{2}}{2 \rho}\right) \\
& -2 \epsilon^{2}\left(\rho-1+\epsilon^{2} \lambda^{2} r^{2}+\epsilon^{4} \lambda_{Z}^{2} z^{2}+V_{0} \exp \left[-\epsilon^{2} r^{2} / r_{l}^{2}\right]\right) \rho=0
\end{aligned}
$$

In particular, for $V_{0}=r_{l}=0.8$ we get the following solution

$$
\rho=\frac{0.18354 r^{2}+0.0910069 r^{4}}{1+0.452004 r^{2}+0.0910069 r^{4}+0.005353 z^{2}} \exp \left(-0.04 r^{2}-0.01 z^{2}\right) .
$$

Figure 3 shows the $r$-dependent plots of (18) and (21) for various values of $z$. The form of the external potential is given as an inset. Notice how the laser beam causes a slight depletion of the condensate close to the centre, followed by an increase in the density elsewhere.

\section{Padé approximations of solitary waves in two dimensions}

So far we have shown that Padé approximations are useful for obtaining accurate approximations of straight line vortices. In this section we obtain approximate solutions of solitary 
Figure 3: Plots of the density function $\rho(r, z)$ at $z=0,5,10,15$ for the vortex solutions with (21) dashed line) and without (18) solid line) the laser beam. The form of the external potential is depicted in the inset.

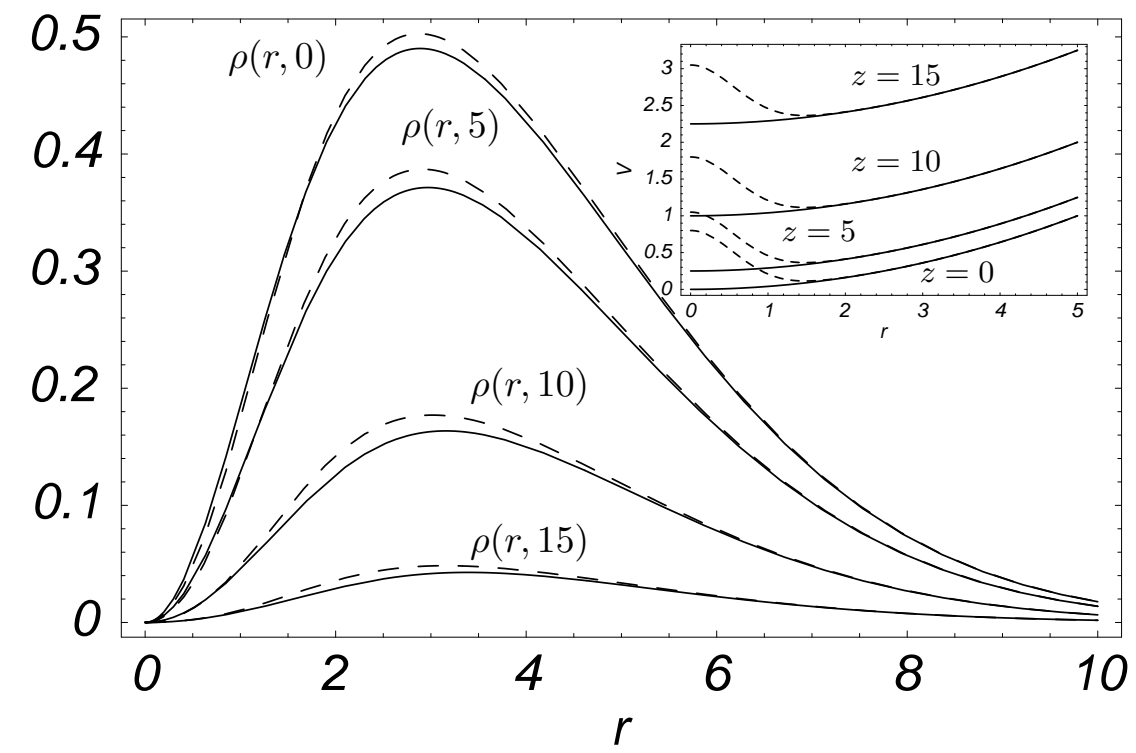

waves moving with a constant velocity. In a seminal paper, Jones and Roberts [10] numerically determined the entire sequence of solitary wave solutions of the GP equation, such as vortex rings, vortex pairs, and rarefaction pulses. Their numerics involved the introduction of stretched variables, expansion of the wave function in double Chebyshev-Legendre series and a Newton-Raphson iteration of the resulting system of nonlinear algebraic equations.

We start with finding Padé approximations of solitary waves in two dimensions (2D). The pair of two point vortices of opposite circulation centred at $\left(0, y_{0}\right)$ and $\left(0,-y_{0}\right)$ with large $y_{0}$ can be approximated by the superposition of wavefunctions of two straight-line vortices of opposite circulation, therefore, by the field with the density

$$
\rho=R^{2}\left(\sqrt{x^{2}+\left(y-y_{0}\right)^{2}}\right) R^{2}\left(\sqrt{x^{2}+\left(y+y_{0}\right)^{2}}\right),
$$

and the phase

$$
S=\arctan \left[\frac{y-y_{0}}{x}\right]-\arctan \left[\frac{y+y_{0}}{x}\right],
$$

where $R(r)$ is given by (5). Figure 4 illustrates how this approximates the solution obtained numerically by a Newton-Raphson iteration of the GP equation

$$
2 i U \frac{\partial \psi}{\partial z}=\nabla^{2} \psi+\left(1-|\psi|^{2}\right) \psi
$$


Figure 4: Plots of the density function $\rho(0, y)$ for vortex pairs with separations $2 y_{0}=8(4 \mathrm{a})$ and $2 y_{0}=1.78$ (4b) taken in a cross-section through the centres of vortices. Solid line - the approximate solution obtained by multiplying the wave functions of individual vortices, dashed line - solution obtained numerically.
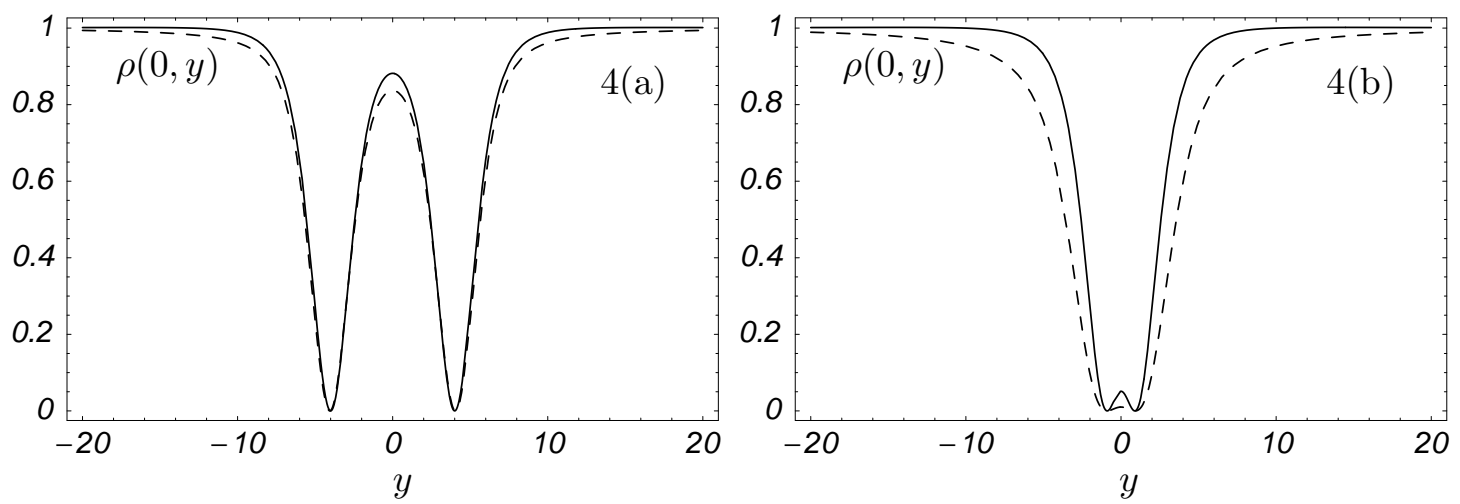

subject to the boundary condition $\psi \rightarrow 1$ as $|\mathbf{x}| \rightarrow \infty$. Here $U$ is the velocity with which the vortices of opposite circulation propel each other in the positive $x$-direction.

The approximation (22)-(23) is sufficiently accurate for large $y_{0}$, but significantly deviates from the numerical solution as the separation between the vortices decreases. Another difficulty in using this approximation is that one needs to know the separation $2 y_{0}$ for each $U$ in order to construct such an approximation.

To obtain Padé approximations of 2D solitary solutions we observe that the approximation (22)-(23) written for the real $u(x, y)$ and imaginary $v(x, y)$ parts of the wavefunction $\psi(x, y)=\sqrt{\rho} \exp [-\mathrm{i} S]=u(x, y)+\mathrm{i} v(x, y)$ are

$$
\begin{aligned}
& u(x, y)=\left(x^{2}+y^{2}-y_{0}^{2}\right) \tilde{R}\left(\sqrt{x^{2}+\left(y-y_{0}\right)^{2}}\right) \tilde{R}\left(\sqrt{x^{2}+\left(y+y_{0}\right)^{2}}\right), \\
& v(x, y)=-2 x y_{0} \tilde{R}\left(\sqrt{x^{2}+\left(y-y_{0}\right)^{2}}\right) \tilde{R}\left(\sqrt{x^{2}+\left(y+y_{0}\right)^{2}}\right)
\end{aligned}
$$

where $\tilde{R}(r)=R(r) / r$. This suggests that a Padé approximation of the solitary wave solution moving in $x$-direction with a constant velocity $U$ can be found in the form

$$
\begin{aligned}
u(x, y) & =\frac{\sum a_{i j} x^{2 i} y^{2 j}}{1+\sum c_{i j} x^{2 i} y^{2 j}}, \\
v(x, y) & =x \frac{\sum b_{i j} x^{2 i} y^{2 j}}{1+\sum c_{i j} x^{2 i} y^{2 j}}
\end{aligned}
$$

We truncate (26) to the lowest order that can give two zeros of the density and unity at 
Figure 5: The equidistant contour and density plots of the density $\rho=u(x, y)^{2}+v(x, y)^{2}$ where $u(x, y)$ and $v(x, y)$ are given by (30). Luminosity is proportional to density, the white area being the most dense.

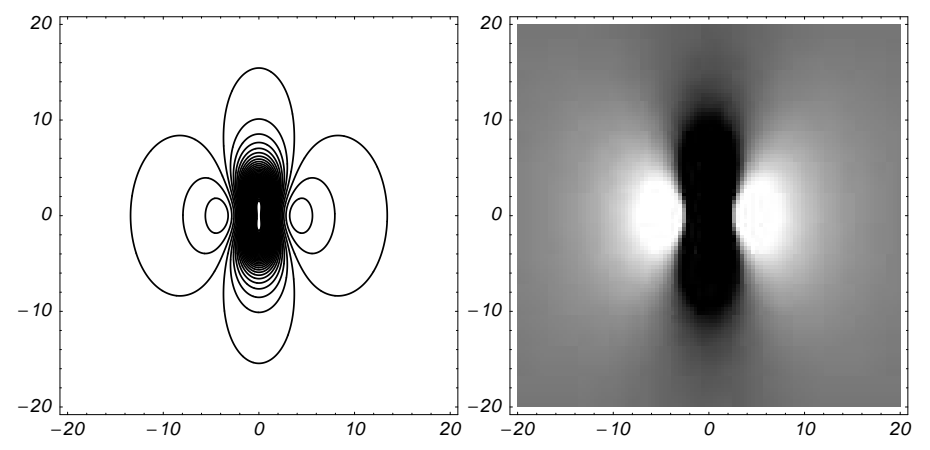

infinity, so we seek an approximation of the form

$$
\begin{aligned}
& u(x, y)=1+\frac{a_{00}+a_{10} x^{2}+a_{01} y^{2}}{1+c_{10} x^{2}+c_{01} y^{2}+c_{20} x^{4}+c_{11} x^{2} y^{2}+c_{02} y^{4}}, \\
& v(x, y)=x \frac{b_{00}+b_{10} x^{2}+b_{01} y^{2}}{1+c_{10} x^{2}+c_{01} y^{2}+c_{20} x^{4}+c_{11} x^{2} y^{2}+c_{02} y^{4}} .
\end{aligned}
$$

We fix the value of $U$, substitute (27) into the equation (24), expand in powers of $x$ and $y$ and set the coefficients at the first three leading orders to zero. As the result we get 12 algebraic equations in 11 variables $a_{i j}, b_{i j}$, and $c_{i j}$ that are compatible and can be solved by a computer algebra. The first two leading orders give the analytical expressions of six coefficients in terms of remaining five. The sum of squares of the remaining six equations is further numerically minimised on the set of remaining five coefficients.

For $U=0.4$ the solution was found as

$$
\begin{aligned}
u & =1+\frac{-1.10048-0.095006 x^{2}+0.01681 y^{2}}{1+0.3108 x^{2}+0.0192 x^{4}+0.1030 y^{2}+0.0219 x^{2} y^{2}+6.112 \times 10^{-3} y^{4}}, \\
v & =\frac{x\left(-0.818647-0.06910 x^{2}-0.03756 y^{2}\right)}{1+0.3108 x^{2}+0.0192 x^{4}+0.1030 y^{2}+0.0219 x^{2} y^{2}+6.112 \times 10^{-3} y^{4}} .
\end{aligned}
$$

The error of the approximation (28) is $\operatorname{Err}_{1} \approx 0.008$, where we defined

$$
E r r_{1}=\max _{x, y}\left(2 U v_{x}+\nabla^{2} u+\left(1-u^{2}-v^{2}\right) u\right)^{2}+\left(-2 U u_{x}+\nabla^{2} v+\left(1-u^{2}-v^{2}\right) v\right)^{2} .
$$

From our constructions the expressions (28) approximate the solution very well for small $x$ and $y$ and give unity for the density at infinity. Two zeros of $u(0, y)$ give the half separation between vortices as 0.893997 (compare it with the value found numerically in [10] as 0.89 to two significant digits). 
We can improve the error $E r r_{1}$, by considering not just the global minimum of the sum of the squares of $\mathcal{O}\left(\epsilon^{4}\right)$ error in the power series expansions about the origin, but also local minima and choosing the one that minimises $E r r_{1}$. This procedure gives us an approximate solution with $\operatorname{Err}_{1}<10^{-4}$ which is

$$
\begin{aligned}
u & =1+\frac{-1.09077-0.0983212 x^{2}-0.00193044 y^{2}}{1+0.31406 x^{2}+0.02026 x^{4}+0.11677 y^{2}+0.02448 x^{2} y^{2}+0.007432 y^{4}}, \\
v & =\frac{x\left(-0.811702-0.0717083 x^{2}-0.0465306 y^{2}\right)}{1+0.314063 x^{2}+0.02026 x^{4}+0.11677 y^{2}+0.024476 x^{2} y^{2}+0.007432 y^{4}} .
\end{aligned}
$$

The energy, $\mathcal{E}$, and momentum, $p$, of solitary wave solutions can be calculated as in [1]:

$$
\begin{aligned}
\mathcal{E} & =\frac{1}{4} \int\left(1-u^{2}-v^{2}\right)\left(3-2 u-u^{2}-v^{2}\right) d x d y, \\
U p & =\frac{1}{2} \int\left(1-u^{2}-v^{2}\right)^{2} d x d y,
\end{aligned}
$$

and are $\mathcal{E} \approx 8.1$ and $p \approx 14.2$ for $u$ and $v$ given by (30). These values can be compared with $\mathcal{E} \approx 8.16$ and $p \approx 14.1$ found numerically in [10. Since we relaxed the precision of the approximation for small $x$ and $y$, the half separation between vortices lost its precision as well, becoming 0.87. Figure 5 shows the contour and density plots of the density $\rho(x, y)=u(x, y)^{2}+v(x, y)^{2}$ of the approximate solution (30).

Finally we consider the third approximation, where we further relax the precision of the approximation at the origin, but impose additional constraints at large $x$ and $y$. The asymptotic solution for large $x$ and $y$ was obtained in [10] as

$$
\begin{aligned}
& u(x, y) \approx 1+\frac{m\left(U-\frac{1}{2} m\right) x^{2}-m U\left(1-2 U^{2}\right) y^{2}}{\left(x^{2}+\left(1-2 U^{2}\right) y^{2}\right)^{2}} \\
& v(x, y) \approx-\frac{m x}{x^{2}+\left(1-2 U^{2}\right) y^{2}}, \quad|\mathbf{x}| \rightarrow \infty
\end{aligned}
$$

where $m$ is a constant termed 'the stretched dipole moment' of the wave because of the factor $1-2 U^{2}$. If we would like our solution to have this asymptotic behaviour at infinity, we have to let

$$
\begin{aligned}
& a_{10}=c_{20} m\left(U-\frac{1}{2} m\right), \quad a_{01}=-c_{20} m\left(1-2 U^{2}\right) U, \quad b_{10}=-m c_{20} \\
& b_{01}=-m c_{20}\left(1-2 U^{2}\right), \quad c_{11}=2 c_{20}\left(1-2 U^{2}\right), \quad c_{02}=c_{20}\left(1-2 U^{2}\right)^{2}
\end{aligned}
$$

The expansion of the equation (24) about zero to $\mathcal{O}\left(\epsilon^{2}\right)$ gives four equations on seven unknowns $U, m, a_{00}, b_{00}, c_{20}, c_{10}, c_{01}$. We can solve these equations analytically for $c_{20}, c_{10}, c_{01}$, and $b_{00}$. In particular, the following approximate relation between the minimum of the real 
Figure 6: Equidistant contour plot of the density $\rho=u(x, y)^{2}+v(x, y)^{2}$ where $u(x, y)$ and $v(x, y)$ are given by (37).

Figure was not included in this submission to keep the size below the maximum allowed.

part of the wavefunction $u(0,0)=a_{00}+1$ and the slope of the imaginary part at the origin in the direction of a solitary motion $v_{x}(0,0)=b_{00}$ was found

$$
v_{x}(0,0)=(u(0,0)-1) \frac{16+m U-20 U^{4}}{7\left(m-4 U^{3}\right)} .
$$

Next we fix one parameter, say $U$, to specify one particular solution of the family, and find $a_{00}$ and $m$ that minimise the integral error

$$
E r r_{2}=\int\left(2 U v_{x}+\nabla^{2} u+\left(1-u^{2}-v^{2}\right) u\right)^{2}+\left(-2 U u_{x}+\nabla^{2} v+\left(1-u^{2}-v^{2}\right) v\right)^{2} d V,
$$

where $d V=d x d y$.

By implementing this procedure, we obtained the following approximation of the vortex pair moving with $U=0.4$

$$
\begin{aligned}
& u(x, y)=1+\frac{-1.14026-0.150112 x^{2}-0.0294564 y^{2}}{1+0.35022 x^{2}+0.03032 x^{4}+0.15905 y^{2}+0.04123 x^{2} y^{2}+0.01402 y^{4}}, \\
& v(x, y)=\frac{x\left(-0.830953-0.108296 x^{2}-0.073641 y^{2}\right)}{1+0.35022 x^{2}+0.03032 x^{4}+0.15905 y^{2}+0.04123 x^{2} y^{2}+0.01402 y^{4}}
\end{aligned}
$$

with the stretched dipole moment found as $m=3.57$ (compared with the value 3.55 found numerically in [10]). The absolute error of this approximation is $E r r_{1} \approx 0.0018$.

In order to find the solitary wave with a single zero of the wave function, we implement the latter procedure by fixing $a_{00}=-1$. This will guarantee that the intersection of zeros of real and imaginary parts is only at the origin. We found that $U=0.45, m=3.32$ and the approximate solution with $E r r_{1}=0.00089$ is

$$
\begin{aligned}
& u(x, y)=1+\frac{-1-0.137233 x^{2}-0.0303092 y^{2}}{1+0.36838 x^{2}+0.03397 x^{4}+0.15803 y^{2}+0.04082 x^{2} y^{2}+0.01227 y^{4}} \\
& v(x, y)=\frac{x\left(-0.803361-0.112913 x^{2}-0.0678506 y^{2}\right)}{1+0.36838 x^{2}+0.03397 x^{4}+0.15803 y^{2}+0.04082 x^{2} y^{2}+0.01227 y^{4}} .
\end{aligned}
$$

On Figure 6 the contour plot of $\rho(x, y)$ is given. This solution is interesting because it represents the borderline case between vortex pair and vortex-free solutions. 
A Padé approximation of a rarefaction pulse can be obtained similarly. For instance, if $U=0.5$ we get $a_{00}=-0.826$ and $m=3.1$, so that the solution is

$$
\begin{aligned}
& u(x, y)=1+\frac{-0.825937-0.114393 x^{2}-0.0271467 y^{2}}{1+0.37355 x^{2}+0.03495 x^{4}+0.14235 y^{2}+0.03495 x^{2} y^{2}+0.008737 y^{4}}, \\
& v(x, y)=\frac{x\left(-0.737901-0.108587 x^{2}-0.0542934 y^{2}\right)}{1+0.37355 x^{2}+0.03495 x^{4}+0.14235 y^{2}+0.03495 x^{2} y^{2}+0.008737 y^{4}} .
\end{aligned}
$$

We will use this approximation of the rarefaction pulse in Section 7 , where we study vortex nucleation.

\section{Generalised rational function approximation of axisym- metric solitary waves in three dimensions}

To determine approximations of the axisymmetric vortex rings and rarefaction pulses moving along the $x$-axis with the constant velocity $U$ in three dimensions (3D), we need to solve (24) with the Laplacian written in cylindrical coordinates

$$
\begin{aligned}
-2 U v_{x} & =u_{x x}+u_{s s}+\frac{1}{s} u_{s}+\left(1-u^{2}-v^{2}\right) u, \\
2 U u_{x} & =v_{x x}+v_{s s}+\frac{1}{s} v_{s}+\left(1-u^{2}-v^{2}\right) v,
\end{aligned}
$$

where $s=\sqrt{y^{2}+z^{2}}$. The asymptotics of the solitary waves at large distances from the origin was found in [10] to have the form

$$
\begin{aligned}
& u(x, s) \approx 1+\frac{2 m U x^{2}-2 m U\left(1-2 U^{2}\right) s^{2}}{\left(x^{2}+\left(1-2 U^{2}\right) s^{2}\right)^{5 / 2}} \\
& v(x, s) \approx-\frac{m x}{\left(x^{2}+\left(1-2 U^{2}\right) s^{2}\right)^{3 / 2}}, \quad|\mathbf{x}| \rightarrow \infty .
\end{aligned}
$$

It is clear that approximation by rational functions can not behave at large distances as (40), therefore, we will use 'generalised' rational functions. In particular, we determined that among many possibilities the following expressions give a reasonable approximation everywhere

$$
\begin{aligned}
& u(x, y)=1+\frac{a_{00}+a_{10} x^{2}+a_{01} s^{2}+m c_{20}^{7 / 4} U\left(2 x^{2}-\left(1-2 U^{2}\right) s^{2}\right)}{\left(1+c_{10} x^{2}+c_{01} s^{2}+c_{20}\left(x^{2}+\left(1-2 U^{2}\right) s^{2}\right)^{2}\right)^{7 / 4}} \\
& v(x, y)=x \frac{b_{00}+b_{10} x^{2}+b_{01} y^{2}-m c_{20}^{7 / 4}\left(x^{2}+\left(1-2 U^{2}\right) s^{2}\right)^{2}}{\left(1+c_{10} x^{2}+c_{01} s^{2}+c_{20}\left(x^{2}+\left(1-2 U^{2}\right) s^{2}\right)^{2}\right)^{7 / 4}}
\end{aligned}
$$

From the two leading order expansions of (39) about the origin we determine $a_{10}, a_{01}, b_{01}, b_{10}$ and the rest of the unknowns will be found by minimising (35), where $d V=s d s d x$, for 
a fixed $U$. Among many local minima that give small $E r r_{2}$, we choose the one with a stretched dipole moment close to the one obtained in [10. In particular, the small vortex ring with $U=0.6$ and $E r r_{1}=0.003$ was found as (41) with

$$
\begin{aligned}
& a_{00}=-1.1, \quad a_{01}=0.0170524, \quad a_{10}=0.0289452, \quad m=8.97 \\
& b_{00}=-0.953, \quad b_{01}=-0.0049767, \quad b_{10}=-0.0594346, \\
& c_{01}=0.04, \quad c_{10}=0.21, \quad c_{20}=0.00612 .
\end{aligned}
$$

We calculate the energy and momentum of the solitary wave solutions in 3D as (see [10])

$$
\begin{aligned}
\mathcal{E} & =\pi \int\left(u_{x}^{2}+u_{s}^{2}+v_{x}^{2}+v_{s}^{2}+\frac{1}{2}\left(1-u^{2}-v^{2}\right)^{2}\right) s d s d x \\
p & =2 \pi \int\left((u-1) v_{x}-v u_{x}\right) s d s d x
\end{aligned}
$$

to get $\mathcal{E} \approx 58.8$ and $p \approx 78$ for (41) with (43), that can be compared with the values found numerically in [10]: $\mathcal{E} \approx 56.4$ and $p \approx 78.9$. The radius of the ring given by (41)-(43) is 1.059 (with 1.06 found numerically).

Finally we give our approximation of the rarefaction pulse moving with $U=0.63$ found on the lower branch of the dispersion curve calculated numerically in [10]:

$$
\begin{aligned}
& a_{00}=-0.79792, \quad a_{01}=0.00388059, \quad a_{10}=0.00882276, \quad m=8.37 \\
& b_{00}=-0.7981, \quad b_{01}=-0.012783, \quad b_{10}=-0.0574092 \\
& c_{01}=0.0399, \quad c_{10}=0.199, \quad c_{20}=0.0058
\end{aligned}
$$

For this approximation of the rarefaction pulse (411)-(44) we have $\mathcal{E} \approx 54.4, p \approx 72.2$, and $m=8.37$ (compared with numerical values found in [10]: $\mathcal{E} \approx 52.3, p \approx 72.2$, and $m=8.37)$.

\section{Vortex nucleation}

The approximations developed in the previous sections allow one to study interactions among the solitary wave solutions of the GP equation. Without an accurate starting point in numerical calculations it would be impossible to separate clearly the effect of interactions from the evolution of each solitary wave by itself as it settled down from a poor initial guess.

In this section we will use the approximations developed above to show that vortex pairs and vortex rings can appear as a result of an interaction among the solitary wave solutions of the GP equation. Previously, the nucleation of vortices in a uniform condensate has been connected to critical velocities [7, 2] or instabilities of the initial states [1]. We will show that interactions between various, even vortex-free, solitary waves result in energy 
Figure 7: [Colour online] The snapshots of the contour plots of the density cross-section of a condensate obtained by numerically integrating the GP model (2). Initial condition is $\psi(t=0)=\Psi(x-5,0) \Psi(x+5,0)$, where $\Psi=u+\mathrm{i} v$, with $u$ and $v$ given by (41)- B4ack solid lines show zeros of real and imaginary parts of $\psi$, therefore, their intersection shows the position of topological zeros. Both low and high density regions are shown in darker shades to emphasise intermediate density regions. Only a portion of an actual computational box is shown.

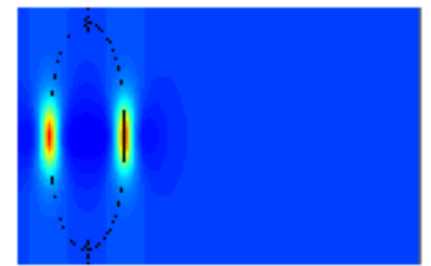

$t=0$

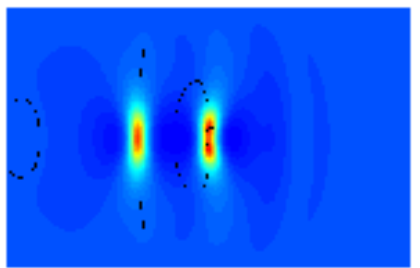

$t=21.6$

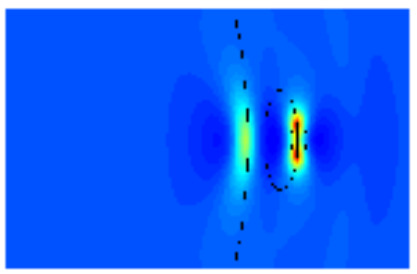

$t=+3.2$

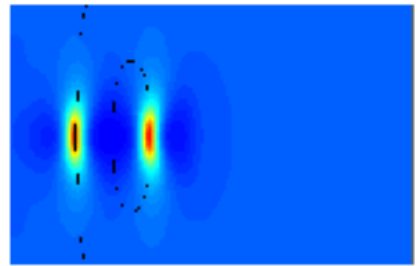

$t=7.2$

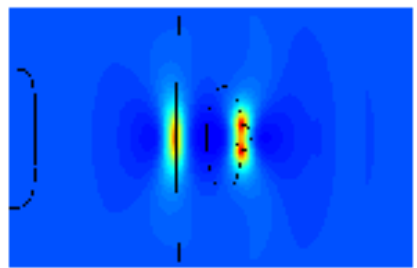

$t=28.8$

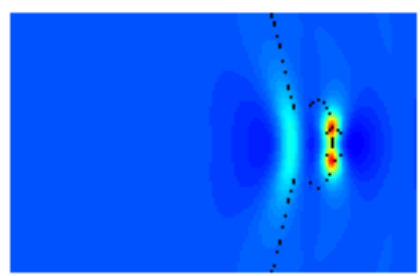

$t=50 . \pm$

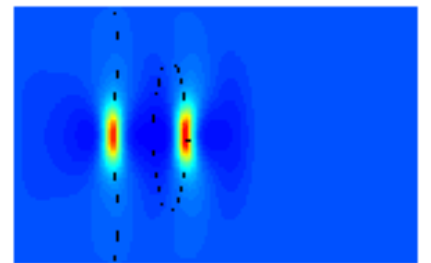

$t=1+.4$

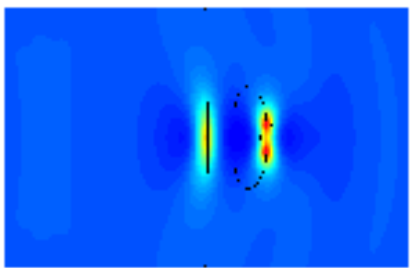

$t=36$

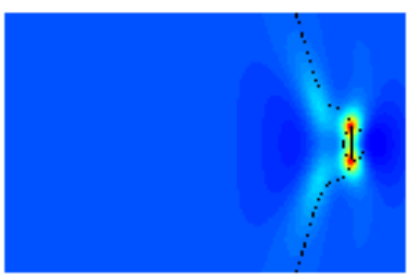

$t=57.6$

and momentum transfer that can lead to vortex nucleation. Rarefaction pulses on the lower branch of the dispersion curve have lower energy and momentum than vortex rings, therefore, such rarefaction pulse may evolve into a vortex ring if interactions with other solutions add enough energy and momentum to the rarefaction pulse.

This scenario is supported by direct numerical simulations, performed with the same numerical method as in our previous work 2, 3. In these computations we follow the evolution of two rarefaction pulses moving in the computational box of dimensions $D^{3}=$ $80^{3}$. The faces of the box are open to allow sound waves to escape; this is achieved numerically by applying the Raymond-Kuo technique [18].

We prepare our initial conditions by superimposing the wavefunctions $\psi_{i}$ of solitary 
Figure 8: [Colour online] The isosurface $\rho / \rho_{\infty}=0.15$ of a condensate. Initial condition is $\psi(t=0)=$ $\Psi(x-5,0) \Psi(x+5,0)$, where $\Psi=u+\mathrm{i} v$, with $u$ and $v$ given by (41)-(44). At $t=0$ the solution is vortex free. AT $t=22.5$ the front pulse contains a closed topological zero. At $t=40$ a well-formed vortex ring has developed with the back solution becoming too shallow to be shown for this isosurface. Only a portion of an actual computational box is shown.
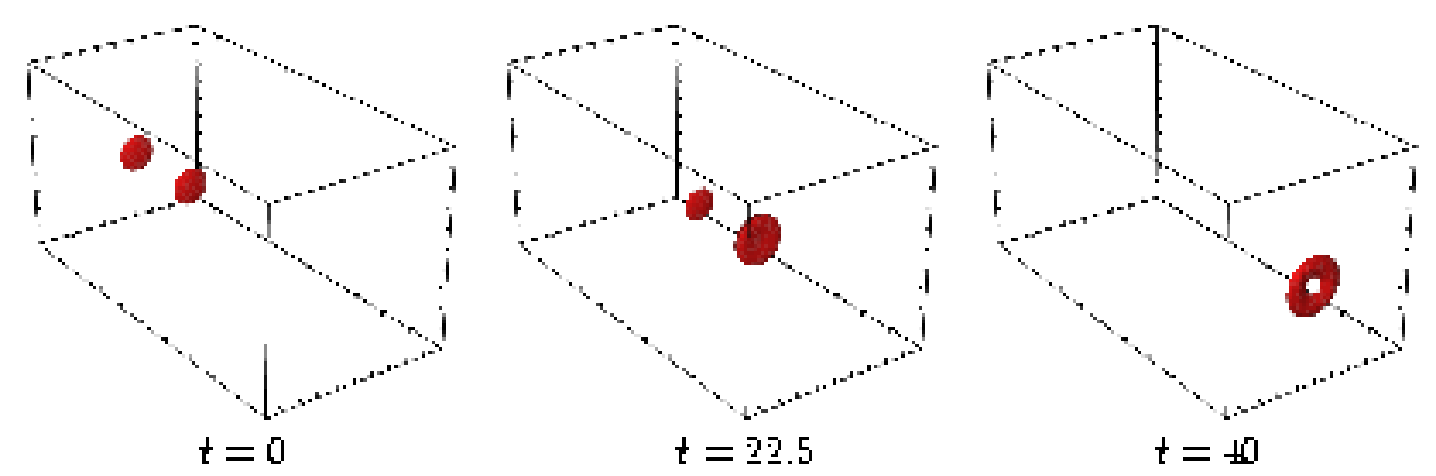

wave solutions of the GP equation found in the previous sections, $\psi(t=0)=\prod \psi_{i}$. If the distance between such solutions is large enough, then such a superposition will not lead to a significant initial sound emission and solitary waves will preserve their form initially. Our first initial state consists of two rarefaction pulses (41)-(44) positioned the distance 10 apart and moving towards each other. Two pulses collide and pass through each other without loss of energy. Next we will create a field nonuniformity by placing two rarefaction pulses a distance 10 apart that move in the same direction. This time the effect two solitary waves have on each other is non-symmetric. As a result, the rarefaction pulse moving behind transfers part of its energy and momentum to the pulse moving at front, so that the latter transforms into a vortex ring and slows down, whereas the former spreads out and speeds up. This process leads to an even closer interaction of the two solitary waves and an even more rapid transfer of energy from the solitary wave that moves behind to the one moving at front. Eventually almost all of the energy and momentum of the former is transferred to the latter, which becomes a vortex ring of energy and momentum that are only slightly less than twice the energy and momentum of each of the initial rarefaction pulses. The remaining small energy is emitted as sound waves. Figure 7 gives the graphical illustration of this process through the snapshots of the density cross-sections. Figure 8 shows the density isoplots at $|\psi|^{2}=0.15$ of the various stages of the ring formation.

Similarly, energy and momentum transfer takes place between different types of solitary waves. In our next calculation we start with a rarefaction pulse followed by a large vortex ring that moves in the same direction. Initially both the distance between these two solitary 
waves and the radius of the ring were taken to be 10 . The rarefaction pulse is moving faster than the vortex ring, so the distance between them rapidly increases. Nevertheless, there is an energy and momentum transfer that allows the rarefaction pulse to evolve into a vortex ring of a small radius and the large vortex ring to shrink slightly. Apart from a small loss of energy to sound waves, the total energy of these two solitary waves is almost conserved throughout this transformation. These processes are shown in graphical form through the contour plots of the density cross-sections (Figure 9) and the density isoplots at $|\psi|^{2}=0.1$. (Figure 10).

Figure 9: [Colour online] The snapshots of the contour plots of the density cross-section of a condensate obtained by numerically integrating the GP model (2). The initial condition is $\psi(t=0)=\Psi_{1}(x, 0) \Psi_{2}(x-$ $5, s+10) \Psi_{2}^{*}(x-5, s-10)$, where $\Psi_{1}=u+\mathrm{i} v$, with $u$ and $v$ given by (41)- (44) and $\Psi_{2}=R\left(\sqrt{x^{2}+y^{2}}\right) e^{\mathrm{i} \theta}$ with $R$ given by (5). Black solid lines show zeros of real and imaginary parts of $\psi$, therefore, their intersection shows the position of topological zeros. Both low and high density regions are shown in darker shades to emphasise intermediate density regions. Only a portion of an actual computational box is shown.

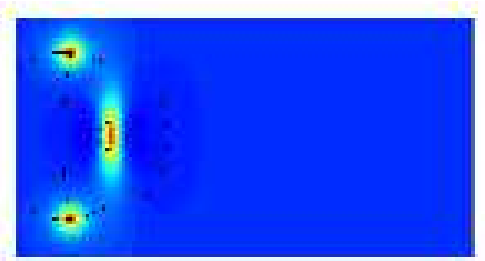

$t=0$

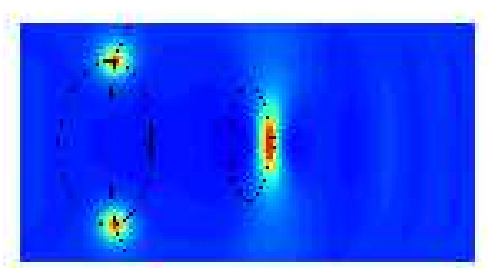

$t=30$

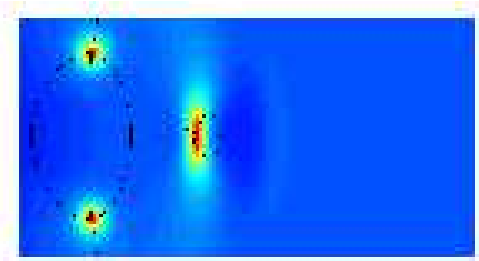

$t=15$

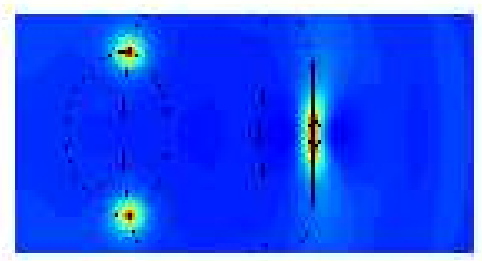

$t= \pm 0$

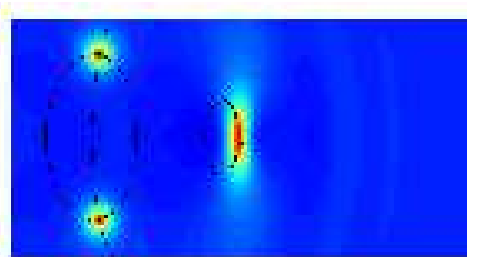

$t=25$

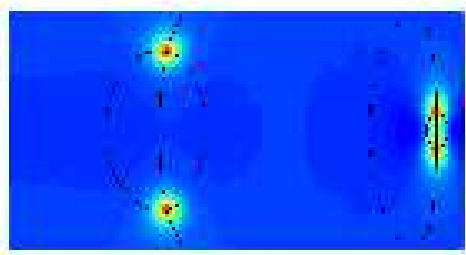

$t=70$

Similar transfer of energy and momentum from one solitary wave to another takes place in 2D. In particular, there is a transfer of energy between solitary waves moving in the same direction, whereas two colliding solitary waves interact elastically. Nevertheless, this elasticity of interactions can be broken by introducing other solitary waves. As an example, we show the evolution of an initial condition consisting of two colliding rarefaction pulses in close vicinity of a widely separated vortex pair. As a result of the interaction, another vortex pair is created and the resulting two pairs of vortices move apart in direction making small angles with the positive $x$ - axis. Figure 11 shows the snapshots of the density of a condensate at various moments of time as the solution evolves. 
Figure 10: [Colour online] The isosurface $\rho / \rho_{\infty}=0.1$ of a condensate. The initial condition is $\psi(t=$ $0)=\Psi_{1}(x, 0) \Psi_{2}(x-5, s+10) \Psi_{2}^{*}(x-5, s-10)$, where $\Psi_{1}=u+\mathrm{i} v$, with $u$ and $v$ given by (41)- (44) and $\Psi_{2}=R\left(\sqrt{x^{2}+s^{2}}\right) e^{\mathrm{i} \theta}$ with $R$ given by (5). At $t=0$ the front pulse is vortex free. AT $t=22.5$ the front pulse contains a closed topological zero. At $t=40$ the front pulse evolved into a well-formed vortex ring. Only a portion of an actual computational box is shown.

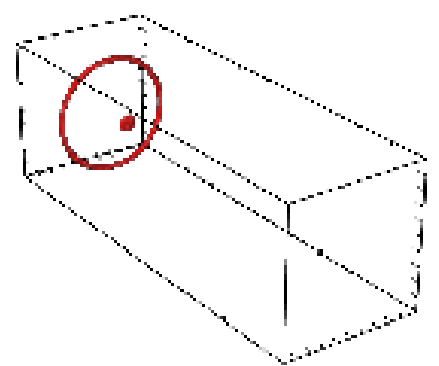

$t=0$

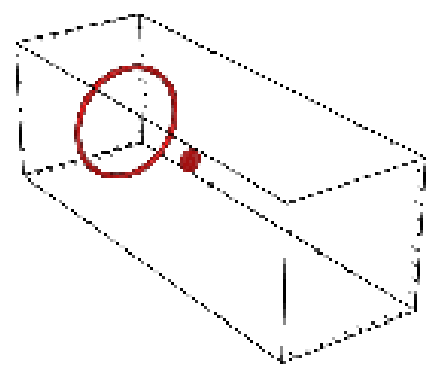

$t=30$

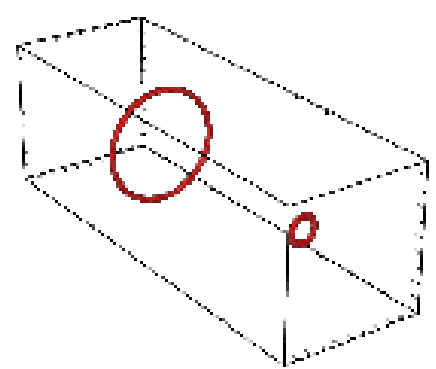

$t=83$

Figure 11: [Colour online] The snapshots of the contour plots of the density of a condensate obtained by numerically integrating the GP model (2) in 2D. Initial condition is $\psi(t=0)=\psi_{1}(x-10,0) \psi_{1}^{*}(x+$ $10,0) \psi_{2}(x, y+4) \psi_{2}^{*}(x, y-4)$, where $\psi_{1}=u+\mathrm{i} v$, with $u$ and $v$ given by (38) and $\psi_{2}=R\left(\sqrt{x^{2}+y^{2}}\right) e^{\mathrm{i} \theta}$ with $R$ given by (5). Black solid lines show zeros of real and imaginary parts of $\psi$, therefore their intersection shows the position of topological zeros. Both low and high density regions are shown in darker shades to emphasise intermediate density regions. Only a portion of an actual computational box is shown.

Figure was not included in electronic submission to keep the size below maximum allowed.

\section{Conclusions}

In summary we have presented a new technique for finding approximate vortex solutions of the GP equation in a uniform condensate and in condensates with axisymmetric traps. These solutions have simple analytic expressions, correct asymptotic behaviours at zero and infinity and approximate the entire solutions quite well elsewhere. We envision that the use of such approximations will allow one to set up accurate initial vortex configurations for numerical calculations and will make explicit analytic manipulations possible.

We have also developed a technique for obtaining approximations of the solitary solutions such as vortex pairs and rarefaction pulses in two and three dimensions. The found approximations are shown to give a very low error and have simple analytical form. These approximations are used to elucidate the energy and momentum transfer between different solitary wave solutions by direct numerical integration of the GP equation. The process of 
vortex nucleation is one of the consequences of such a transfer.

\section{Acknowledgements}

The author is grateful to Professor Paul Roberts for useful discussions and comments about this manuscript. This work is supported by the NSF grant DMS-0104288.

\section{References}

[1] Berloff N G 2002 Phys. Rev. B 65174518

[2] Berloff N G and Roberts P H 2000 J. Phys. A: Math. Gen. 334025

[3] Berloff N G and Roberts P H 2001 J. Phys. A: Math. Gen. 3410057

[4] Caradoc-Davies B M, Ballagh R J and Burnett K 1999 Phys. Rev. Lett 803903

[5] Donnelly R J 1991 Quantized Vortices in Helium II (Cambridge: Cambridge University Press)

[6] Fetter A L and Svidzinsky A A 2001 J. Phys.: Condens. Matter 13 R135

[7] Frisch T, Pomeau Y and Rica S 1992 Phys. Rev. Lett. 691644

[8] Ginzburg V L and Pitaevskii L P 1958 Zh. Eksp. Teor. Fiz. 341240

[9] Gross E P 1963 J. Math. Phys. 4195

[10] Jones C A and Roberts P H 1982 J. Phys. A: Gen. Phys. 152599

[11] Jones C A, Putterman S J and Roberts P H 1986 J. Phys. A: Mathe. Gen. 192991

[12] Konotop V V and Perez-Garcia V M 2000 Phys. Rev. A 62033610

[13] Koplik J and Levine H 1993 Phys. Rev. Lett. 71 1375; 1996 Phys. Rev. Lett. 764745

[14] Leadbeater M, Winiecki T, Samuels D C, Barenghi C F, and Adams C S 2001 Phys. Rev. Lett. 861410

[15] Nazarenko S and West R 2003 J. Low Temp. Phys., 1321

[16] Nore C, Abid M and Brachet M E 1997 Phys. Fluids 92644

[17] Pitaevskii L P 1961 Sov. Phys. JETP 13451 
[18] Raymond G W and Kuo H L 1984 Q J R Meteorol Soc 110525

[19] Roberts P H and Grant J 1971 J. Phys. A: Gen. Phys. 455

[20] Staliunas K 2000 Appl. Phys. B 71555 